\title{
Ulnar neuropathy after forearm fracture in a child: the role of peripheral nerve ultrasound in the decision of management
}

\author{
Timur Ekiz, ${ }^{1}$ Hanifi Üçpunar, ${ }^{2}$ Davut Yıldız ${ }^{2}$ \\ ${ }^{1}$ Department of Physical Medicine and Rehabilitation, Elbistan State Hospital, Kahramanmaraş, Turkey \\ ${ }^{2}$ Department of Orthopedics and Traumatology, Elbistan State Hospital, Kahramanmaraş, Turkey
}

Received: April 08, 2017 Accepted: June 19, 2017 Published online: July 14, 2017

A 13-year-old boy was consulted from the Department of Orthopedics to our rehabilitation clinic for ultrasonographic evaluation of the ulnar neuropathy -whether the nerve was intact and a nerve repair was required- one month after a forearm fracture. In his medical history, he was admitted to the emergency department after a fall and was diagnosed with as a left forearm fracture one month previously (Figure 1). After closed reduction was performed, radius and ulna fractures were fixated with titanium elastic nail by using mini open incision technique.

Physical examination revealed claw hand deformity, weakness in the abductor digiti minimi muscle, intrinsic muscle atrophy, and numbness on the ulnar side in his left hand. Electrodiagnostic testing was consistent with ulnar neuropathy. For the underlying pathology, X-rays were non-remarkable. Ultrasound clearly showed the ulnar nerve (enlarged and hypoechoic bundles due to edema, but intact) and fracture line. Besides, ultrasonographic evaluation revealed a positive sono-Tinel sign at the level of injury. No bony spur or scar tissue was detected. The nerve was not entrapped, and the surrounding tissues were intact (Figure 2). Control visits were recommended to avoid a redo surgery for the ulnar nerve. He had no motor dysfunction and his symptoms improved after a three-month rehabilitation program.

Ulnar nerve palsy has been reported as a rare complication of closed mid-shaft forearm fractures. ${ }^{[1,2]}$ Direct contusion/damage by a bone spike or entrapment by a bone spike/scar can be the underlying mechanisms. The ulnar nerve runs under the deep layer of the flexor carpi ulnaris muscle on the forearm and is protected by the surrounding muscle. ${ }^{[1,2]}$ Therefore, direct contusion by an external force is a rare condition. Nonetheless, the nerve lies closed to the ulna at the middle and distal thirds; therefore significant angulation and displacement with a spike can directly damage the nerve. ${ }^{[1,2]}$

In the treatment, a neurolysis/exploration is required in case of any entrapment. Although similar cases have been reported in the literature, it is difficult to uniform treatment (as surgical indications, the time of surgery time, etc.). ${ }^{[1,2]}$ To the best of our knowledge, the role of musculoskeletal ultrasound in the previous cases has not been highlighted. Accordingly, with the presented case, we would like to highlight that ultrasound is a

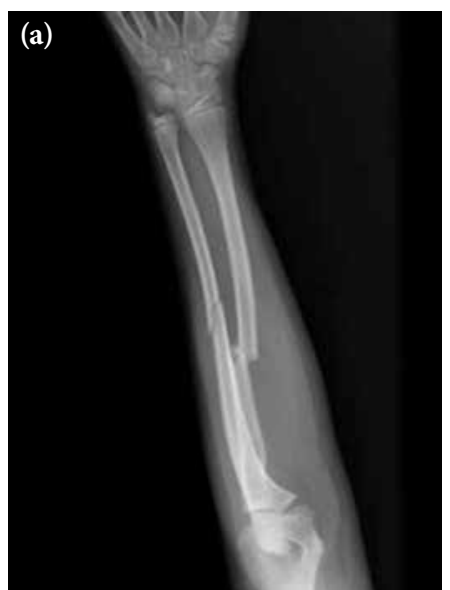

Figure 1. (a) Preoperative and showing radius and ulna fractures.

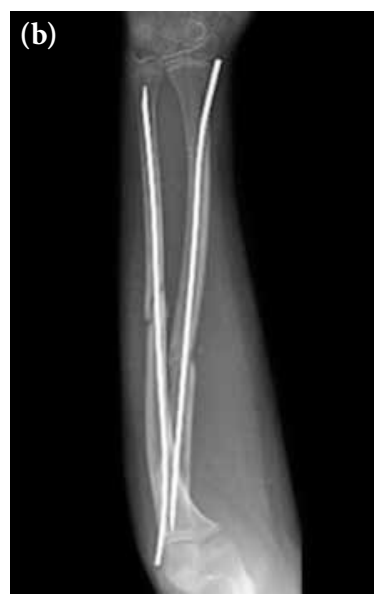

(b) postoperative X-rays 

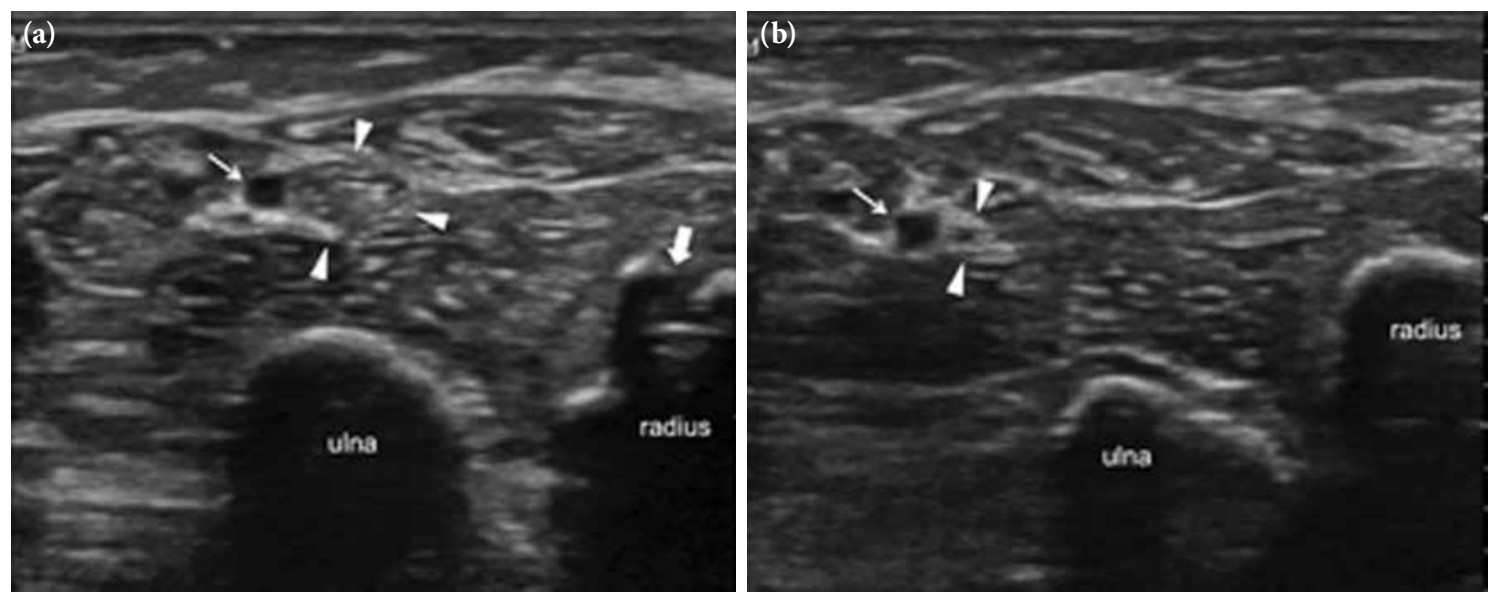

Figure 2. Ultrasound image (a) designates the ulnar nerve (enlarged and hypoechoic bundles due to edema, but intact) (arrow heads) nearby the ulnar artery (thin arrow) and fracture line (thick arrow). No bony spur or scar tissue was detected. The nerve was not entrapped, and surrounding tissues were intact. Right image (b) illustrates the ulnar nerve as normal just proximal to the level of injury.

convenient imaging modality to visualize peripheral nerves and guide for the management of nerve insults with several advantages (i.e., ease of application, cost-effective, widely available, sono-Tinel, provides dynamic visualization). ${ }^{[3]}$

\section{Declaration of conflicting interests}

The authors declared no conflicts of interest with respect to the authorship and/or publication of this article.

\section{Funding}

The authors received no financial support for the research and/or authorship of this article.

\section{REFERENCES}

1. Suganuma S, Tada K, Hayashi H, Segawa T, Tsuchiya H. Ulnar nerve palsy associated with closed midshaft forearm fractures. Orthopedics 2012;35:e1680-3.

2. Hirasawa H, Sakai A, Toba N, Kamiuttanai M, Nakamura $\mathrm{T}$, Tanaka K. Bony entrapment of ulnar nerve after closed forearm fracture: a case report. J Orthop Surg (Hong Kong) 2004;12:122-5.

3. Kara M, Özçakar L, De Muynck M, Tok F, Vanderstraeten G. Musculoskeletal ultrasound for peripheral nerve lesions. Eur J Phys Rehabil Med 2012;48:665-74. 